\title{
BLOOD FLOW UNDER EXTERNAL STRAINS: PHENOMENOLOGICAL APPROACH, THEORETICAL DEVELOPMENTS AND NUMERICAL ANALYSIS
}

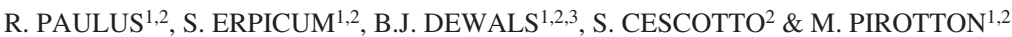 \\ ${ }^{1} \mathrm{HACH}$ (Hydrology, Applied Hydrodynamics and Hydraulic Constructions), University of Liège, Belgium. \\ ${ }^{2}$ ArGEnCo (Architecture, Geology, Environment and Constructions), University of Liège, Belgium. \\ ${ }^{3}$ F.R.S - FNRS (Belgian Fund for Scientific Research), Belgium.
}

\begin{abstract}
In the medical field, the measurement of blood flow characteristics is often necessary. More specifically, blood pressure is an essential measure when it comes to assessing health. All over the world, many people suffer from hyper- or hypotension, and as it is known that these diseases can lead to serious complications, it is of great interest to determine the blood pressure with high accuracy. Nowadays, such information requires the use of specific materials; the present method for the measurement of the arterial pressure, by applying pressure using an armband (with a control device called sphygmomanometer), is known to introduce significant errors due to the inadequacy of the band dimensions (both the length and the circumference). The objective of the present research is to study and simulate the discharge of the blood in an artery subjected to external strains using theoretical developments and a numerical approach. Based on these modelling results, the response of the fluid to the external pressure of the band can be studied, and finally appropriate corrective factors for the true pressure and the measured pressure could be assessed. This research has been carried out with the aim of sharing medical and engineering views on the subject. The artery can be modelled as a deformable pipe, where the blood flowing in it is a fluid with specific properties. Thus, two complementary and interconnected domains are covered, solid mechanics (to obtain analytic relations between the strains and the deformations, using either linear or non-linear theories) and fluid mechanics (to study the discharge of blood in a deformable pipe, using finite volume methods), therefore considering the problem as a loose fluid-structure interaction (FSI). These two domains, which are well studied for common materials in civil engineering applications, are applied here not only to specific materials but especially to uncommon structures that, besides the somehow common FSI developments, lead to the investigation and research of very specific boundary conditions, giving them a physically based behaviour. At present, the research has reached the penultimate step, with the two main mentioned axes being fully developed and tested on their own. In particular, the boundary conditions developed for the models have been investigated and modelled in depth.

Keywords: $1 D$ numerical flow modelling, capillary behaviour, (non)-linear material analysis, original outflow boundary conditions.
\end{abstract}

\section{INTRODUCTION}

Blood pressure is by far one of the most essential measures when it comes to assessing human health. Although it is well known that hyper- and hypotension diseases are widespread all over the world, one does not always know the impact of a physiologically abnormal measure. For instance, an increase of $5 \mathrm{mmHg}$ will double the risks of cardiovascular diseases; it is therefore essential not to underestimate the blood pressure. It is also important not to overestimate it because that would lead to unnecessary treatments which are costly and sometimes risky if the patient is in good health.

The principal method used to measure blood pressure at present is based on Korotkoff's [1] and Riva-Rocci's [2] discoveries and developments and it follows a well-established medical procedure [3]. Although very widespread all over the world, this technique is known to induce potentially significant errors in the measurement, and modern medical approaches are currently being developed in order to avoid the use of this technique [4-6]. At our unit, the objective has been to simulate this operating mode, postulating that a numerical approach to the phenomenon would enhance the accuracy of the common surgical appliance. 
For many years, fluid-structure interaction (FSI) has been widely used in order to simulate blood propagation in vessels, and, more generally, to study biomechanics as well as haemodynamic problems [7-9]. The interaction between the fluid and the surrounding structure can vary from loose to strong, and so the coupling between the two models follows the same scheme; interactions with materials of high deformability should be modelled using strong coupling [10, 11], whereas interactions with rigid materials can be modelled using loose coupling [12-14]. The method chosen in our unit is based on a strong interaction that is required here due to the strong deformability of the vessels. The mechanical behaviour is taken into account in a direct way in the flow modelling, using an ingenious modelling device called the Preismann slot for this purpose.

This coupled approach is somehow classical in this field $[7-9,15,16]$, but the way the boundary conditions are represented still remains a challenge. Indeed, most of the time, the downstream boundary condition is linked to a pressure signal. But, since this signal is, in a way, the unknown that our research focuses on, it is rather awkward to consider it fixed and known downstream. Therefore, physically based boundary conditions have been identified, thus avoiding the imposition of a downstream signal.

The solution method is explained briefly in Section 2. Then the two independent models representing, respectively, the solid mechanics and the fluid mechanics are presented and discussed in Sections 3 and 4 , before the boundary conditions are discussed at the end of the latest. Finally, the results obtained for the boundary condition developments are presented, analysed and discussed in Section 5.

\section{SOLUTION METHOD}

The goal of this research is to simulate the discharge of the blood in the arteries, more specifically when the arteries are subjected to external strains, as is the case during blood pressure measurement.

The artery can be modelled as a deformable pipe, where the blood flowing in it is a fluid with specific properties which is pulsed into the artery. The cardiovascular system has a specific architecture and structure that requires specific boundary conditions. Thus, the research involves two complementary and interconnected domains, namely solid mechanics and fluid mechanics; therefore, the FSI problem of interest here is the simulation of the discharge of a specific fluid into a network of deformable pipes. The analytic relations between the external strains (not only the armband pressure but also the blood pressure) and the deformations can be obtained using either linear or non-linear theories. In this manner, the way the artery changes its shape may be determined. Given the mathematically known deformations for the artery, the discharge of the blood in this deformable pipe, comprising vessels with a very wide range of diameters, has been studied based on the WOLF modelling system, fully developed at the University of Liège.

\section{MECHANICAL ANALYSIS OF THE ARTERY}

The first part of the research focuses on solid mechanics. As mentioned before, hydraulic modelling requires knowing the way in which the artery deflates under specific strains (be it internal or external pressures). The analytic relations between the section and the pressures have been derived. First, the necessary hypothesis are presented, followed by a detailed description of the mathematical developments.

\subsection{Specificities of the artery}

The arm is a complex material composed of muscles, bones, vessels and tendons among others.

Although a complete study of the limb could be undertaken (by means of numerical modelling), analytic equations are sought here. Therefore, assumptions are needed, both about the geometry and 
about the mechanical behaviour of the material. The arm is considered to be composed of two coaxial materials, namely the artery and the muscle, and the contacts between them have been neglected, thus assuming that the pressures are uniformly applied (cf. Fig. 1a). Besides, it is known that the materials are subject to long deformations and that they can be assumed to be elastic materials.

To obtain the analytic results, two additional hypotheses are necessary, one concerning the material properties and the other concerning the material's behaviour under strain. The muscle and the artery are supposed to be transversely isotropic and to follow a plane state of strain. These two assumptions are supported by observations about the material's structure, as discussed in previous papers [17-19]; the structures of both the artery and the muscle are assumed to be concentric (and so present a cylindrical symmetry), the stresses are perpendicular to the solids generatrix, and one can reasonably accept that the longitudinal extensions are insignificant (the vessels are confined axially).

\subsection{Mathematical analysis}

As the artery and the muscle are viewed as a two-material coaxial pipe, one can develop the problem using the cylindrical coordinate and following the law of the solid mechanics.

Following the previous assumptions, a non-linear analysis is obviously required. However, for now, only the theoretical developments of this analysis as well as the implementation of a numerical model based on the mathematical results have been done. The last step would be to calibrate this model in order to make it usable in the final model.

The linear analysis, based on the more restrictive assumption that the displacement is relatively small, has been done in a similar manner and is already available since the parameters of the model are mostly available in literature [20-22].

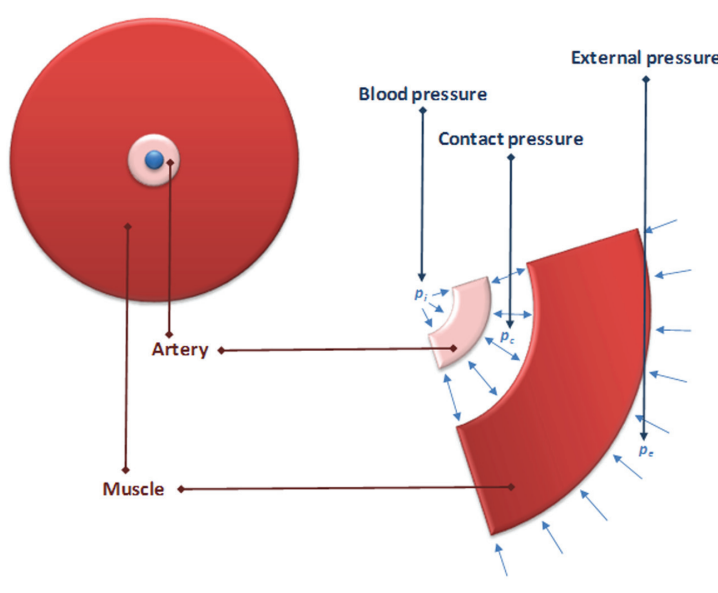

(a)
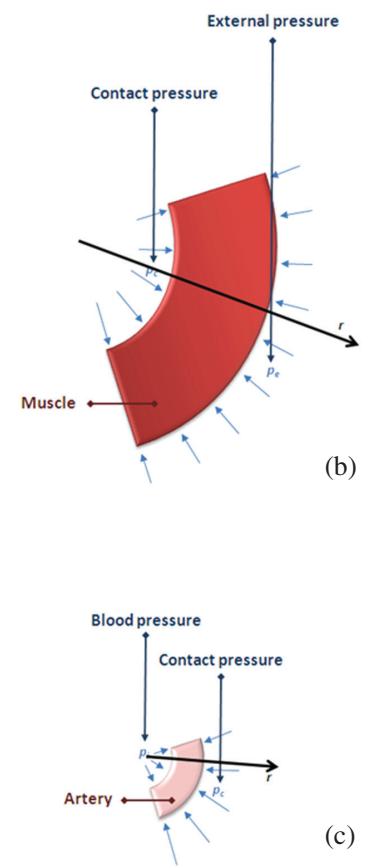

Figure 1: (a) Transverse section of the arm, idealized situation; (b) muscle with external and contact pressures; and (c) artery with contact and internal pressures. 


\subsubsection{Linear analysis}

The linear analysis gives the first approach to the problem, and the different developments are rather obvious. Successively, the two cylinders are considered to be subjected to the concerned pressures (e.g. when the muscle has been considered, the internal and contact pressures have been taken into account), and the displacements are expressed as a function of the respective pressures (see Fig. 1b and c).

Finally, by setting the displacements at the contact zone as equal, an analytic relation is obtained for the section of the artery, function of the different materials (Hooke's parameters for the artery and the muscle), the dimensions and the pressures $[17,18]$. The results are shown below and represent the radial displacement for the artery (eqn (1)), the equilibrium contact pressure (eqn (2)) and the section (eqn (3)).

$$
\begin{gathered}
u_{r}\left(r=r_{i}\right)=\frac{1}{2} \frac{1}{G_{1}}\left(\frac{1}{r_{c}^{2}-r_{i}^{2}}\right)\left(\left(1-2 v_{1}\right)\left(r_{i}^{3} p_{i}-r_{c}^{2} r_{i} p_{c}^{x}\right)+\left(p_{i}-p_{c}\right) r_{i} r_{c}^{2}\right), \\
p_{c}=2 \frac{\frac{1}{G_{1}}\left(\frac{1}{r_{c}^{2}-r_{i}^{2}}\right)\left(1-v_{1}\right) r_{i}^{2} p_{i}+\frac{1}{G_{2}}\left(\frac{1}{r_{e}^{2}-r_{c}^{2}}\right)\left(1-v_{2}\right) r_{e}^{2} p_{e}}{\left(\frac{1}{G_{2}}\left(\frac{1}{r_{e}^{2}-r_{c}^{2}}\right)\left(r_{c}^{2}\left(1-2 v_{2}\right) r_{e}^{2}\right)+\frac{1}{G_{1}}\left(\frac{1}{r_{c}^{2}-r_{i}^{2}}\right)\left(r_{c}^{2}\left(1-2 v_{1}\right)+r_{i}^{2}\right)\right)}, \\
\Omega=\pi\left(r_{i}+u_{r}\left(r=r_{i}\right)\right)^{2},
\end{gathered}
$$

where $u_{r}[\mathrm{~m}]$ is the axial distortion; $r[\mathrm{~m}]$ is the radial position; $r_{e}, r_{i}$ and $r_{c}[\mathrm{~m}]$ are, respectively, the external, internal and contact radii; $G_{1}$ and $G_{2}[\mathrm{~Pa}]$ are, respectively, the artery and muscle bulk modulus; $v_{1}$ and $v_{2}[-]$ are, respectively, the artery and muscle Poisson coefficients; $p_{e}, p_{i}$ and $p_{c}[\mathrm{~Pa}]$ are, respectively, the external, internal and contact pressures; $\Omega\left[\mathrm{m}^{2}\right]$ is the section. This set of equations is valid only under the assumptions detailed in Section 3.1.

\subsubsection{Non-linear analysis}

A Mooney-Rivlin function, which is one of the most simple and comprehensive strain energy density functions, has been chosen to represent the hyperelastic material. It takes the form of $W=$ $C_{1}\left(I_{1}-3\right)+C_{2}\left(I_{1}-3\right)$, where $I_{1}[\mathrm{~m}]$ and $I_{2}[\mathrm{~m}]$ are the first and second invariant of the deviatoric component of the Finger tensor and $C_{1}[\mathrm{~Pa}]$ and $C_{2}[\mathrm{~Pa}]$ are parameters inherent to the materials.

The equations obtained [17] are presented below:

$$
\begin{gathered}
u_{r}=r-R, \\
r=\sqrt{\frac{R^{2}+C(1+k)}{1+k},}
\end{gathered}
$$

$$
\begin{aligned}
A^{x}= & -q_{B} \\
& -\frac{R_{B}^{2}\left[2 C_{1}(1+k)+2 C_{2}+2 C_{2}(1+k)^{3}+\left[C_{1}+C_{2}(1+k)^{2}\right](1+k)\left\{\frac{C(1+k)}{R_{B}^{2}+C(1+k)}+2 \ln R_{B}-\ln \left(R_{B}^{2}+C(1+k)\right)\right\}\right]}{(1+k)^{2}\left(R_{B}^{2}+C(1+k)\right)} \\
& +\frac{2 C_{2} C(1+k)+C(1+k)^{2}\left(C_{1}+C_{2}(1+k)^{2}\right)\left(\frac{C(1+k)}{R_{B}^{2}+C(1+k)}+2 \ln R_{B}-\ln \left(R_{B}^{2}+C(1+k)\right)\right)}{\ldots},
\end{aligned}
$$




$$
\begin{aligned}
& \sigma_{r}\left(R=R_{A}\right)=-q_{A} \\
& =\frac{R_{A}^{2}\left[2 C_{1}(1+k)+2 C_{2}+2 C_{2}(1+k)^{3}+\left(A^{x}+\frac{\left[C_{1}+C_{2}(1+k)^{2}\right]}{1+k}\left\{\frac{C(1+k)}{R_{A}^{2}+C(1+k)}+2 \ln R_{A}-\ln \left(R_{A}^{2}+C(1+k)\right)\right\}\right)(1+k)^{2}\right]}{(1+k)^{2}\left(R_{A}^{2}+C(1+k)\right)} \\
& +\frac{2 C_{2} C+\left(A^{x}+\frac{\left[C_{1}+C_{2}(1+k)^{2}\right]}{1+k}\left\{\frac{C(1+k)}{R_{A}^{2}+C(1+k)}+2 \ln R_{A}-\ln \left(R_{A}^{2}+C(1+k)\right)\right\}\right) C(1+k)^{2}}{(1+k)\left(R_{A}^{2}+C(1+k)\right)}, \\
& \Omega=\pi\left(r_{A}\right)^{2},
\end{aligned}
$$

where $u_{r}[\mathrm{~m}]$ is the axial displacement, $r[\mathrm{~m}]$ is the radius position after distortion, $R[\mathrm{~m}]$ is the radius position before distortion, $R_{A}$ and $R_{B}[\mathrm{~m}]$ are, respectively, the internal and external radii, $C$ $[\mathrm{Pa}]$ and $A^{x}[\mathrm{~Pa}]$ are integrative constants (determined from the boundary behaviour), $\Omega\left[\mathrm{m}^{2}\right]$ is the section, $q_{A}$ and $q_{B}[\mathrm{~Pa}]$ are, respectively, the internal and external pressures, $C_{1}[\mathrm{~Pa}]$ and $C_{2}[\mathrm{~Pa}]$ are parameters inherent to the materials, $k[-]$ is a factor of longitudinal distortion and $\sigma_{r}[\mathrm{~Pa}]$ is the radial constraint.

These equations need some explanations about their signification and use. Equations (4), (5) and (8) are obvious and, when combined, give the section function of $C$ and $k, k$ being zero in plane state of strain. The two integrative constants are given considering the boundary behaviour through eqns (6) and (7).

The presented equations apply to one material considered individually, and the complete resolution must thus be iterative in order to give a stable solution (the contact zone is then taken as the equilibrium zone, and the goal of the iterative method is to balance the relative displacements of this zone). This set of equations is also only valid under the assumptions introduced in Section 3.1.

\subsection{Results and discussion}

The established equations give the response of both the artery and the muscle to the external strains, but the material parameters have not yet been calibrated from the comprehensive set of experiments. The geometrical and mechanical parameters of the materials make up the data of the model. The pressures are the input parameters, and the output data are the radial displacement, from which the section can be obtained. The initial condition consists of a situation without any external constraint. The model evaluates the displacements at the contact zone for both materials, and iterates until they are independent of the considered material, with an error of $0.05 \%$ on the relative displacement.

The results presented here (Figs 2 and 3) involve a comparison of both models for parameters representing the same materials, thus enabling the assessment of the sensitivity of the model.

The presented results correspond to the following data [17]: $r_{i}=1,950 \mu \mathrm{m}, r_{c}=2,950 \mu \mathrm{m}, r_{e}=$ $1,950 \mu \mathrm{m}, E_{\text {Mat1 }}=1.7 \mathrm{MPa}, v_{\text {Mat1 }}=0.5, E_{\text {Mat2 }}=0.003 \mathrm{MPa}, v_{\text {Mat2 }}=0.5, C_{1, \text { Mat1 }}=0.159 \mathrm{MPa}$, $C_{2, \text { Mat } 1}=0.116 \mathrm{MPa}, C_{1, \text { Mat2 }}=0.015 \mathrm{MPa}, C_{2, \text { Mat2 }}=0.007 \mathrm{MPa}$.

The analytic developments presented in this section are based on realistic assumptions (Section 3.1), and the models may already be used in a number of applications. The next step will be to calibrate the models and to further analyse their sensitivity and assumed validity to simulate the physical behaviour of the arm during blood pressure measurement. 


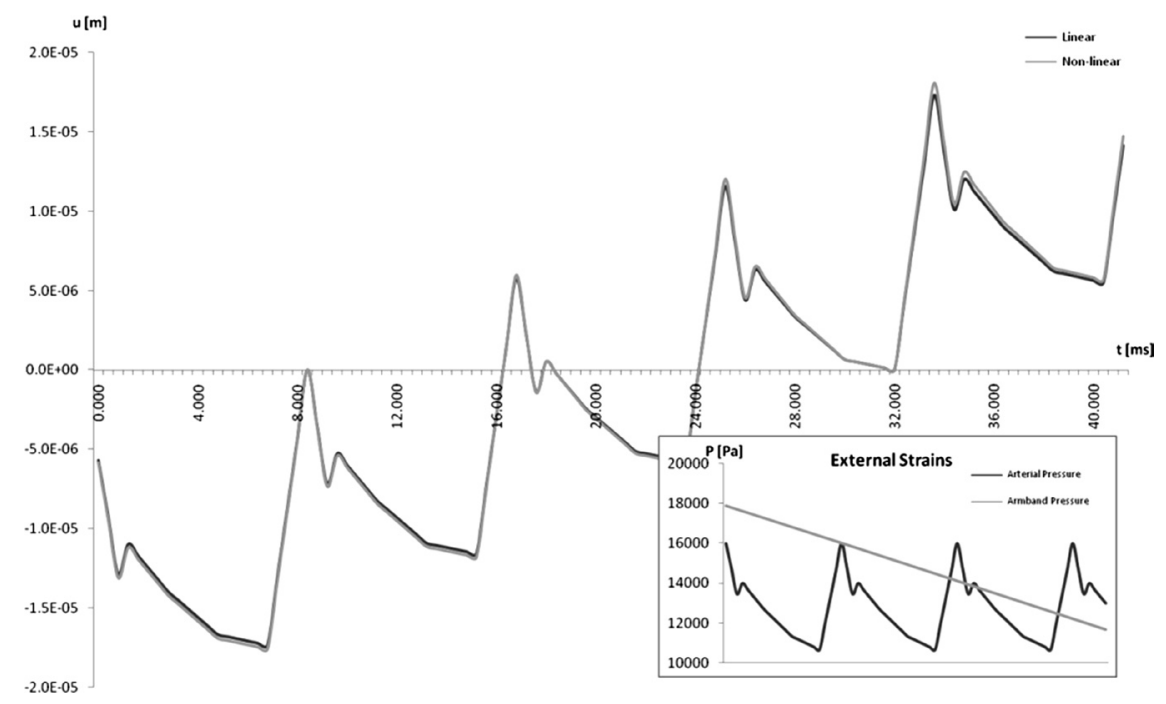

Figure 2: Evolution of the radial displacements [m] due to external strain [Pa], as a function of time.

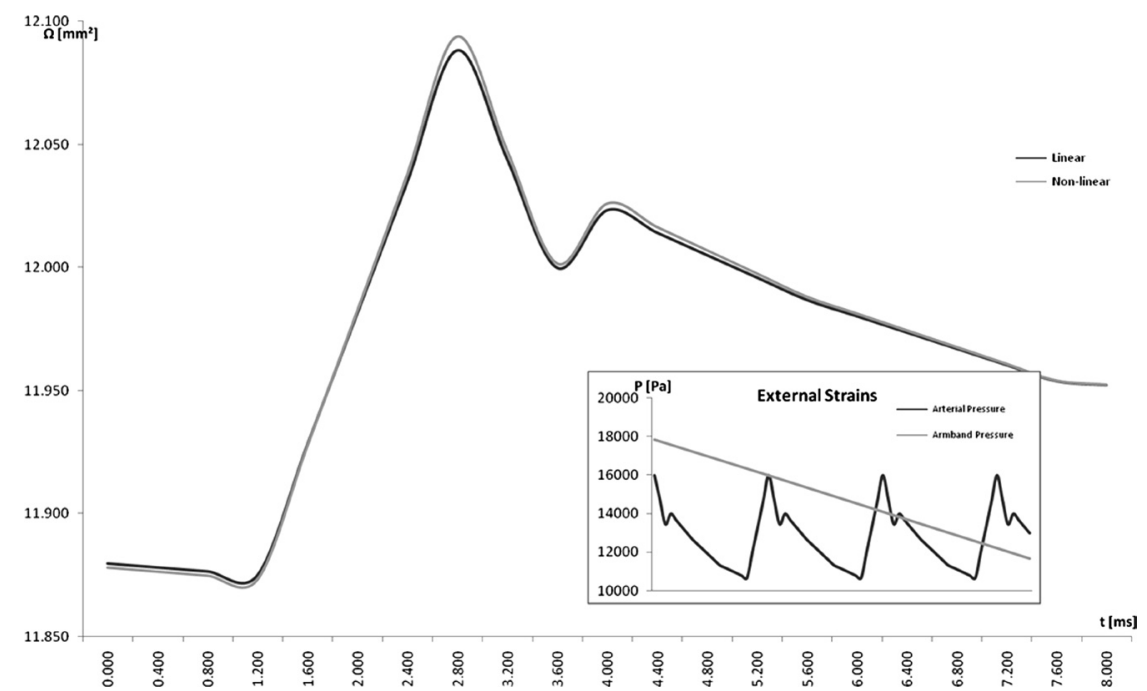

Figure 3: Evolution of the section $\left[\mathrm{mm}^{2}\right]$ due to external strain $[\mathrm{Pa}]$, as a function of time.

\section{HYDRAULIC ANALYSIS OF THE FLOW IN THE ARTERY}

This part of the research is dedicated to the study of the blood discharge in the artery, modelled as the particular deformable pipe described above.

First, the characteristics of blood have to be investigated. Then, the mathematical and numerical developments are outlined based on these assumptions.

While all these developments are common for hydraulic phenomena, Section 4.4, concerning the boundary conditions, will present an original method, as well as its results, developed in order to model the downstream boundary conditions of the artery as a result of the behaviour of the fluid in the capillary network. 


\subsection{Characteristics of blood}

Blood can be distinguished from other fluids based on its viscosity, determined by the haematocrit, and it is obviously a non-Newtonian fluid, but can acceptably be assumed as incompressible [23]. Blood behaves like a Bingham fluid, with its viscosity being a function of the velocity gradient. The latter is thus often expressed as a relative viscosity, being the ratio of the blood viscosity to the plasma viscosity, which is Newtonian. However, in large vessels, the fluid can be considered as Newtonian and homogeneous [24, 25], as the boundary layer is very small compared to the vessel radius. In the capillaries, this assumption is no longer acceptable, the flow being determined mainly by viscous stresses. Besides, it can reasonably be assumed that blood is incompressible, the Mach number being globally quite small. Finally, the turbulent effects have been neglected. It is well known that some sources of instabilities create local turbulence, but analysis of both Reynolds and Womersley numbers shows that this last hypothesis is acceptable [23]. For the smaller vessels, however, these assumptions have to be reconsidered, but for now they have not been updated, and it has been considered that the properties of the fluid remains constant in the whole domain, independent of the vessel dimensions.

\subsection{Mathematical model}

The equations governing the fluid mechanics problem are the Navier-Stokes equations:

$$
\left\{\begin{array}{l}
\frac{\partial \rho}{\partial t}+\nabla(\rho \cdot U)=0 . \\
\frac{\partial U}{\partial t}+U \cdot \nabla U=\frac{-1}{\rho} \nabla p+v \cdot \nabla^{2} U .
\end{array}\right.
$$

They represent, respectively, the conservation of the mass and the momentum. The two variables are the density $(\rho)\left[\mathrm{kg} / \mathrm{m}^{3}\right]$ and the velocity $(U)[\mathrm{m} / \mathrm{s}]$, with respect to the time $(t)[\mathrm{s}]$ and the coordinates $x, y$ and $z[\mathrm{~m}]$. The other parameters in this system are the pressure $(p)[\mathrm{Pa}]$ and the kinematic viscosity (v) $\left[\mathrm{m}^{2} / \mathrm{s}\right]$.

In the specific framework of blood flow, the flow is mostly one-dimensional (1D) and the crosssectional velocities are small. In addition, despite the fact that the streamline curvature cannot actually be considered as small, no serious mistakes are made if these are neglected. With these three acceptable assumptions in mind, the Navier-Stokes equations can be area-integrated [26] into the following form:

$$
\begin{aligned}
& \left\{\begin{array}{l}
\frac{\partial \Omega}{\partial t}+\frac{\partial Q}{\partial x}=q_{l}, \\
\frac{\partial Q}{\partial t}+\frac{\partial}{\partial x}\left(\frac{Q^{2}}{\Omega}+g^{*} I_{1}\right)=g^{*} I_{2}+\frac{F_{x}}{\rho}+U q_{l},
\end{array}\right. \\
& \left\{\begin{array}{l}
I_{1}(h)=\int_{-h_{b}}^{h_{s}}(h-\xi) l(x, \xi) d \xi \\
I_{2}(h)=\int_{-h_{b}}^{h_{s}}(h-\xi) \frac{\partial l(x, \xi)}{\partial x} d \xi
\end{array}\right.
\end{aligned}
$$


where $x[\mathrm{~m}]$ is the axial position, $\Omega\left[\mathrm{m}^{2}\right]$ is the section, $Q\left[\mathrm{~m}^{3} / \mathrm{s}\right]$ is the discharge, $q_{l}\left[\mathrm{~m}^{2} / \mathrm{s}\right]$ is the lateral in- or outflow, $g^{*}\left[\mathrm{~m} / \mathrm{s}^{2}\right]$ is the projected gravity acceleration, $F_{x}\left[\mathrm{~kg} / \mathrm{s}^{2}\right]$ is the global effect of roughness, $h_{b}[\mathrm{~m}]$ is the bottom height, $h_{s}[\mathrm{~m}]$ is the slot height.

The Preismann slot model [27] allows the modelling of pressurized flow using equations similar to those for free surface flows. It consists of a narrow slot on the top of a closed pipe [28, 29].

The width of the slot $\left(T_{f}[\mathrm{~m}]\right)$ is chosen in order to represent the behaviour of the conceptual free surface flow, with a gravity wave speed $(c)[\mathrm{m} / \mathrm{s}]$ represented by

$$
c=\sqrt{\frac{g^{*} \Omega}{T_{f}}}, \quad \frac{1}{c^{2}}=\frac{\rho}{\Omega} \frac{\partial \Omega}{\partial p},
$$

where $c[\mathrm{~m} / \mathrm{s}]$ is the wave speed. The section $\Omega$ and its derivative with respect to the pressure $p$ refer to the law of solid mechanics developed above.

\subsection{Numerical resolution}

The set of non-linear equations introduced above (cf. Section 4.2) requires the use of a numerical resolution. As for many of the hydraulic applications, a finite volume method is an appropriate way to solve the problem.

The set of equations can be written as follows:

$$
\frac{\partial}{\partial x} \boldsymbol{U}+\frac{\partial}{\partial x} \boldsymbol{F}(\boldsymbol{U})=\boldsymbol{S}(\boldsymbol{U})
$$

where the vectors of conservative variables and sources are, respectively,

$$
\boldsymbol{U}=\left(\begin{array}{l}
\Omega \\
Q
\end{array}\right), \quad \boldsymbol{S}(\boldsymbol{U})=\left(\begin{array}{c}
q_{l} \\
g^{*} I_{2}+\frac{F_{x}}{\rho}+U q_{l}
\end{array}\right), \quad \boldsymbol{F}(\boldsymbol{U})=\left(\begin{array}{c}
Q \\
\frac{Q^{2}}{\Omega}+g^{*} I_{1}
\end{array}\right) .
$$

The flux is computed using a flux-vector splitting [30], involving fractioning the flux into two distinct components

$$
\boldsymbol{F}(\boldsymbol{U})=\boldsymbol{F}^{+}\left(\boldsymbol{U}_{l}\right)+\boldsymbol{F}^{-}\left(\boldsymbol{U}_{\boldsymbol{r}}\right)
$$

where $\boldsymbol{U}_{\boldsymbol{l}}$ and $\boldsymbol{U}_{\boldsymbol{r}}$ are the reconstructed value of the conservative variables at the edge of the mesh, respectively, built at the left and right edges. This division takes the following form:

$$
\boldsymbol{F}^{+}=\left\{\begin{array}{l}
\boldsymbol{F}^{\text {up }}, \\
\boldsymbol{F}^{\text {down }},
\end{array} \quad \boldsymbol{F}^{-}=\left\{\begin{array} { l } 
{ \boldsymbol { F } ^ { \text { down } } , } \\
{ \boldsymbol { F } ^ { \text { up } } , }
\end{array} \quad \text { if } \left\{\begin{array}{l}
\boldsymbol{Q} \geq 0, \\
\boldsymbol{Q}<0,
\end{array}\right.\right.\right.
$$

where $Q$ is the characteristic value of the speed at the interface between two meshes, and ( $\left.F^{\text {up }}, F^{\text {down }}\right)$ are the relative fluxes for variables taken, respectively, up- and downstream:

$$
\boldsymbol{F}^{\text {up }}=\left\{\begin{array}{l}
Q, \\
\varphi Q^{2} / \Omega,
\end{array} \quad \boldsymbol{F}^{\text {down }}=\left\{\begin{array}{l}
0 . \\
g I_{1} .
\end{array}\right.\right.
$$

Finally, the integration of eqn (13) leads to the following conservative formulation:

$$
\boldsymbol{U}_{i}^{n+1}=\boldsymbol{U}_{i}^{n}-\Delta t\left[\frac{\boldsymbol{F}_{i+1 / 2}-\boldsymbol{F}_{i-1 / 2}}{\Delta x}+\boldsymbol{S}\right] \text {. }
$$


The numerical resolution uses a three-step Runge-Kutta time-integration scheme, with the three following substep evaluations $(k=1,2,3$, with $k=0$ corresponding to the value of the vectors of conservative variables at step $n$ ):

$$
\boldsymbol{U}_{i}^{n, k}=\boldsymbol{U}_{i}^{n}-\Delta t\left[\frac{\boldsymbol{F}_{i+1 / 2}\left(\boldsymbol{U}^{n, k-1}\right)-\boldsymbol{F}_{i-1 / 2}\left(\boldsymbol{U}^{n, k-1}\right)}{\Delta x}+\boldsymbol{S}\left(\boldsymbol{U}_{i}^{n, k-1}\right)\right] .
$$

And the final solution is given by

$$
\boldsymbol{U}_{i}^{n+1}=R K_{1} \boldsymbol{U}_{i}^{n, 1}+R K_{2} \boldsymbol{U}_{i}^{n, 2}+R K_{3} \boldsymbol{U}_{i}^{n, 3},
$$

with $\sum_{\mathrm{i}} \mathrm{RK}_{\mathrm{i}}=1$.

This numerical model has been fully developed within the HACH unit. Its robustness and ability to handle a very wide range of problems has been shown, tested and successfully validated in many steady as well as unsteady cases [31].

\subsection{Boundary conditions}

The numerical resolution requires the prescription of boundary conditions. The hydraulic conditions observed in this specific case require the forcing of the upstream discharge and the downstream pressure. While it is acceptable to assume that the upstream discharge can be estimated with some accuracy at the upstream of the brachial artery, for instance around the shoulder [22, 32, 33], this is not the case for the downstream quantity. Indeed, the downstream boundary condition is linked to a pressure signal. But, since this signal is the unknown that our research focus on, it may not be considered as fixed and known downstream.

By contrast, we exploit the fact that the behaviour of both velocities and pressures is known in the capillaries. The pressure decays and stabilizes in the capillary network of blood flowing to the veins $[18,19]$, whereas the velocity tends to zero. Moreover, the capillary network presents a somewhat fractal structure with very dense ramifications.

The idea therefore is to impose downstream boundary conditions as capillary conditions so that the imposition of a constant low pressure, at the end of a certain ramified structure, can be done using branches following the architecture of vessels in the arm.

Along the artery, punctual pumping is considered, in order to represent the local branches bringing the blood to the veins through the capillary network, schematized as in Fig. 4a. In this network, the equations of flow are the same as those presented before (4.2.1), but, since only one way is

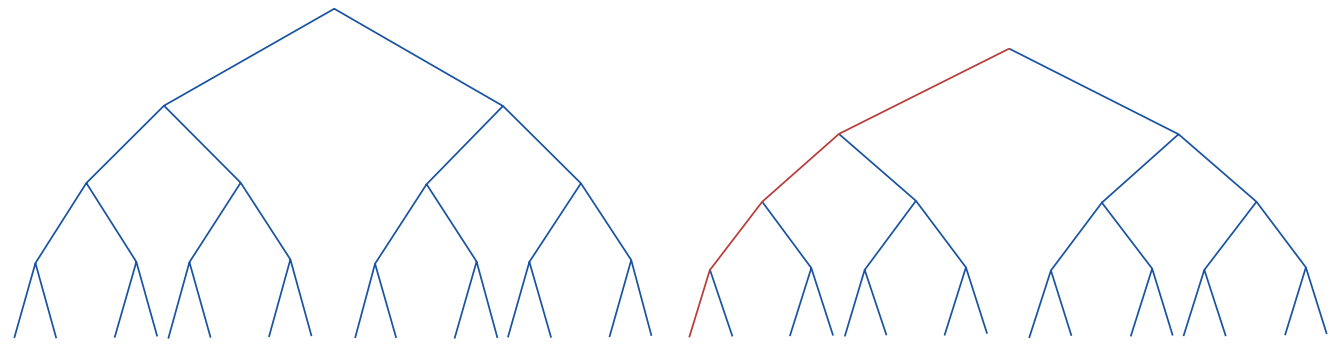

(a)

(b)

Figure 4: Schematization of the capillary network as modelled in the capillary flow: (a) global scheme and (b) one way considered. 
considered (dashed line in Fig. 4b), the fluxes are re-evaluated at each junction, in order to obtain the true discharge to the capillaries.

For this approach to remain valid, the assumptions concerning blood rheology should still hold, which is questionable for the non-Newtonian effects. Nevertheless, since the purpose of modelling the capillary network is to obtain boundary conditions for the artery flow, using a pumping law that will be calibrated, it is reasonable to assume the behaviour as Newtonian, as a preliminary step to a physically based boundary condition.

\section{RESULTS AND DISCUSSION}

For now, the final model coupling both the structure and the fluid model is yet to be built. Nevertheless, the innovative developments in the boundary conditions can be tested and discussed. The remaining developments required before reaching the last step are well known in the current researches on FSI. Therefore, we focus here on the specific results concerning the so-called boundary model.

First, the boundary conditions are modelled on their own, with the aim of assessing their effectiveness. Next, their effect as a downstream boundary condition is compared with more classical outflow conditions, in order to judge the reliability of the original method that is presented in this paper.

The behaviour of the flow is influenced by two main parameters: the slot width and the friction coefficient. The slot represents, through its relation with the wave speed, the deformability of the pipe (see eqn (12)). For instance, a small wave speed (obtained with a relatively high slot width) leads to the representation of a deformable material for the pipe.

If these two parameters are appropriately chosen, the required dampening and the known drop can be well represented. Examples of this process have already been discussed in Ref. [19], and appropriate parameter values have been discussed in the same paper.

The results shown in Figs 5-7 illustrate the evolution of both the dimensional discharge and the dimensional head along the axial position of a single capillary and the evolution of the head as a

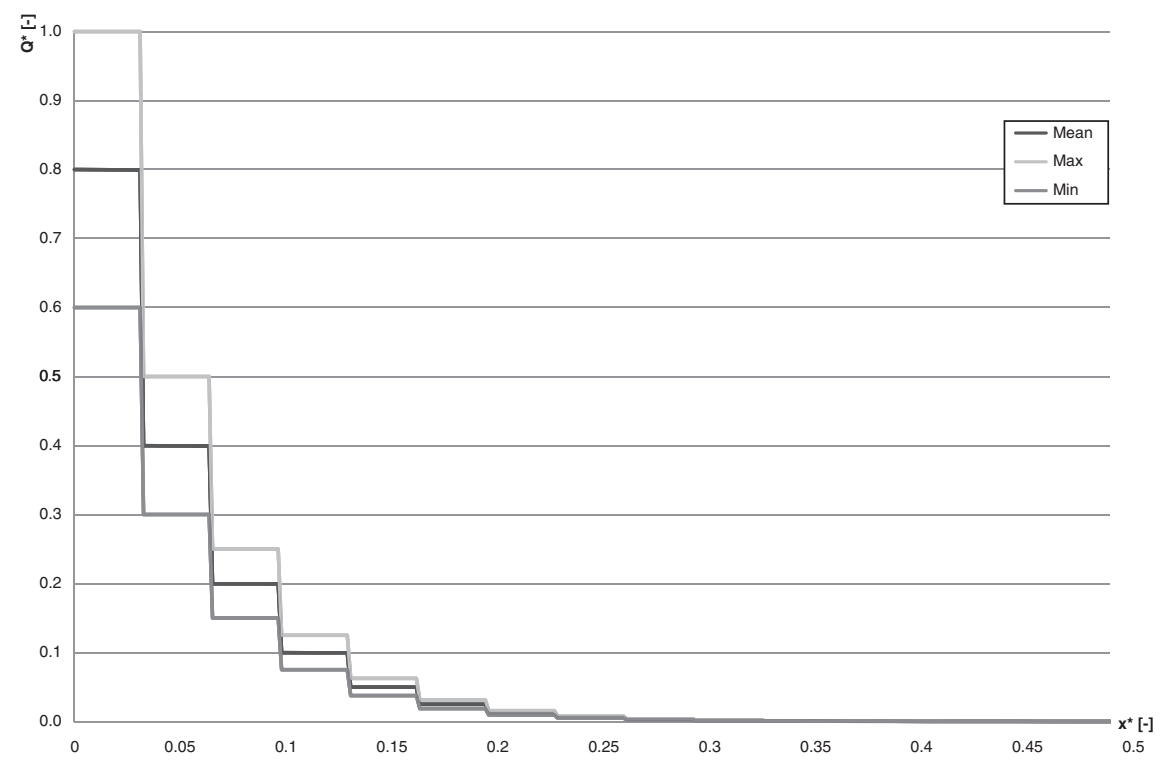

Figure 5: Evolution of the dimensional discharge [-] as a function of the axial position [-]. 


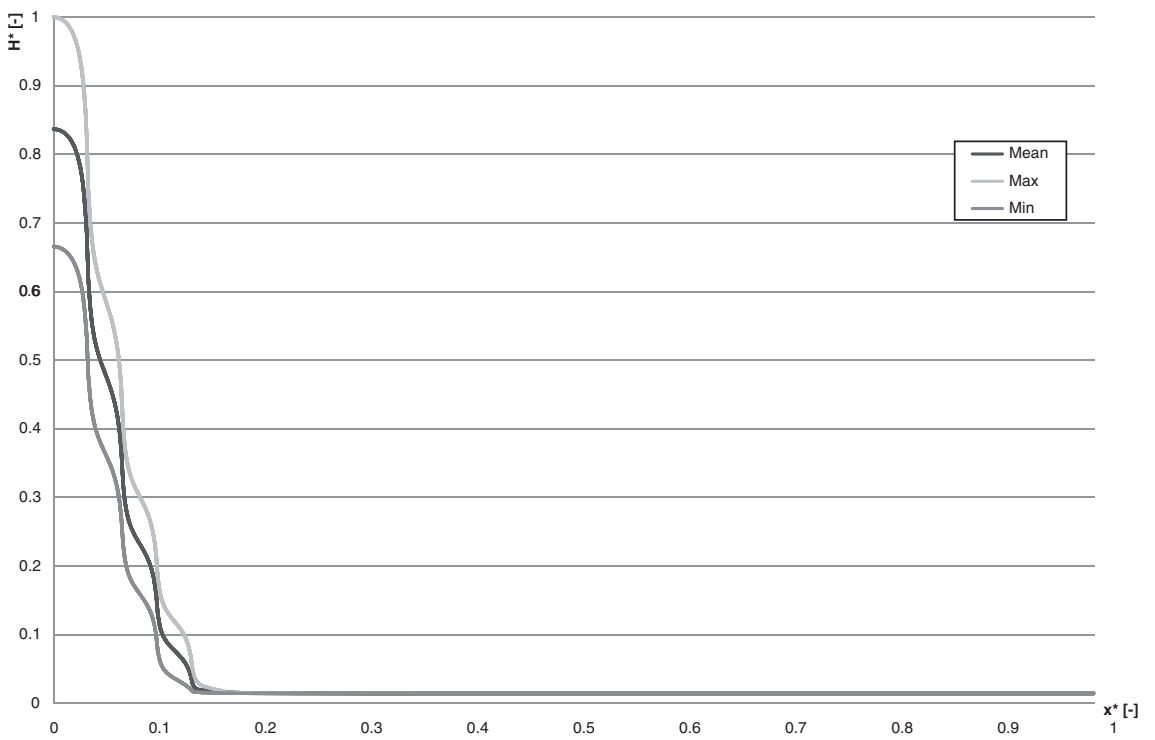

Figure 6: Evolution of the dimensional head [-] as a function of the axial position [-].

function of time. In Fig. 5, the evolution of the dimensional discharge as a function of the axial position is depicted, for three different times corresponding, respectively, to the maximum, the minimum and the mean value of the sinusoidal signal. In Fig. 6, the same technique is used to represent the evolution of the head as a function of the axial position.

In Fig. 7, the evolution of the dimensional head as a function of time is depicted at different locations: successively at the upstream location of the network and at five different branches, from the first to the fifth branch.

The presented results correspond to the following numerical data: $R K_{1}=0.15, R K_{2}=0.45, R K_{3}=$ 0.4. The fluid characteristics are the following: $\rho=1,025 \mathrm{~kg} / \mathrm{m}^{3}, \mu=3.2 \mathrm{cP}$ for a haematocrit of $40-45 \%$. The boundary conditions used for these simulations are indeed a constant pressure at the downstream position of the capillary network, combined with a sinusoidal upstream discharge signal, which enables, even if it is a long way from the read signal, interpreting the influence of the parameters and visualizing the required effects.

It is effectively clear that the consideration of a wide capillary subdivision combined with appropriate parameters representing the dissipative behaviour of the materials lead to the observation of the required dampening pretty quickly.

With these observations in mind, it remains now to assess the reliability of the proposed method as a boundary condition. For this purpose, a simple case of a prismatic pipe is simulated using two different types of outflow boundary conditions, namely our original proposition and the more basic concept of peripheral resistance, as proposed among others by Anliker et al. [34]. Both concepts rely on the fact that the terminal pressure is assumed to be constant, after vanishing in the capillaries. More comparisons are possible, but due to the lack of validation and the uncompleted integration of the whole model at the present time, it has been decided that the validation compared to the most basic and common downstream boundary condition was a first significant step available for an objective comparison. Once our model is finally integrated and calibrated, it will be time to compare it with existing more advanced models. 


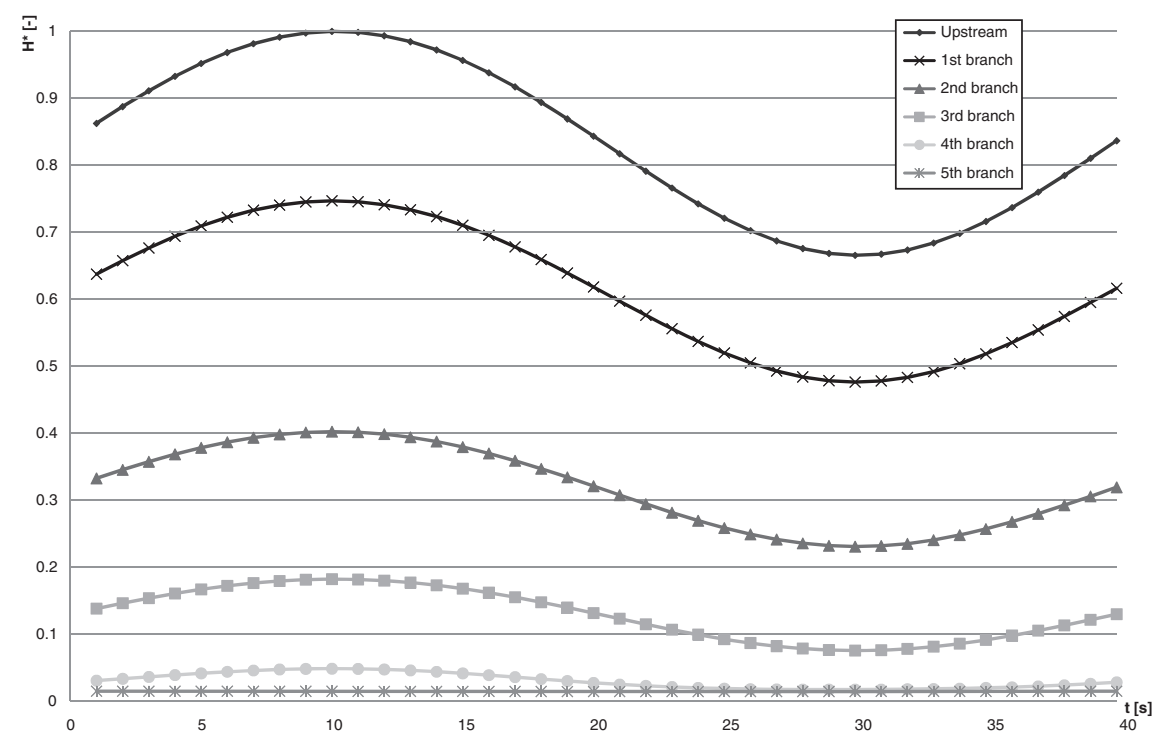

Figure 7: Evolution of the dimensional head [-] as a function of the time [s] at the very upstream of different branches.

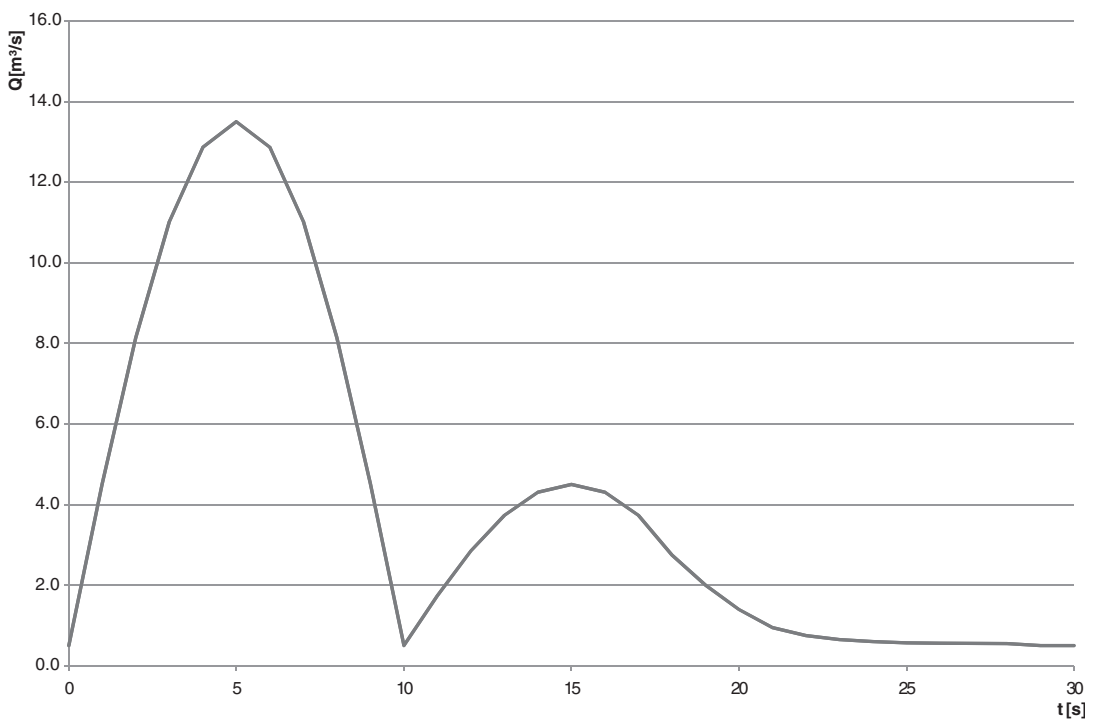

Figure 8: Evolution of the upstream discharge $\left[\mathrm{m}^{3} / \mathrm{s}\right]$ as a function of time [s].

The upstream boundary condition consists of a discharge signal, as presented in Fig. 8 .

Besides, the initial condition is the situation corresponding to a uniform flow of $0.5 \mathrm{~m}^{3} / \mathrm{s}$. The results are presented in Fig. 9 as: (a) model with a peripheral resistance as the downstream boundary condition; (b) model with a capillary network as the downstream boundary condition. 

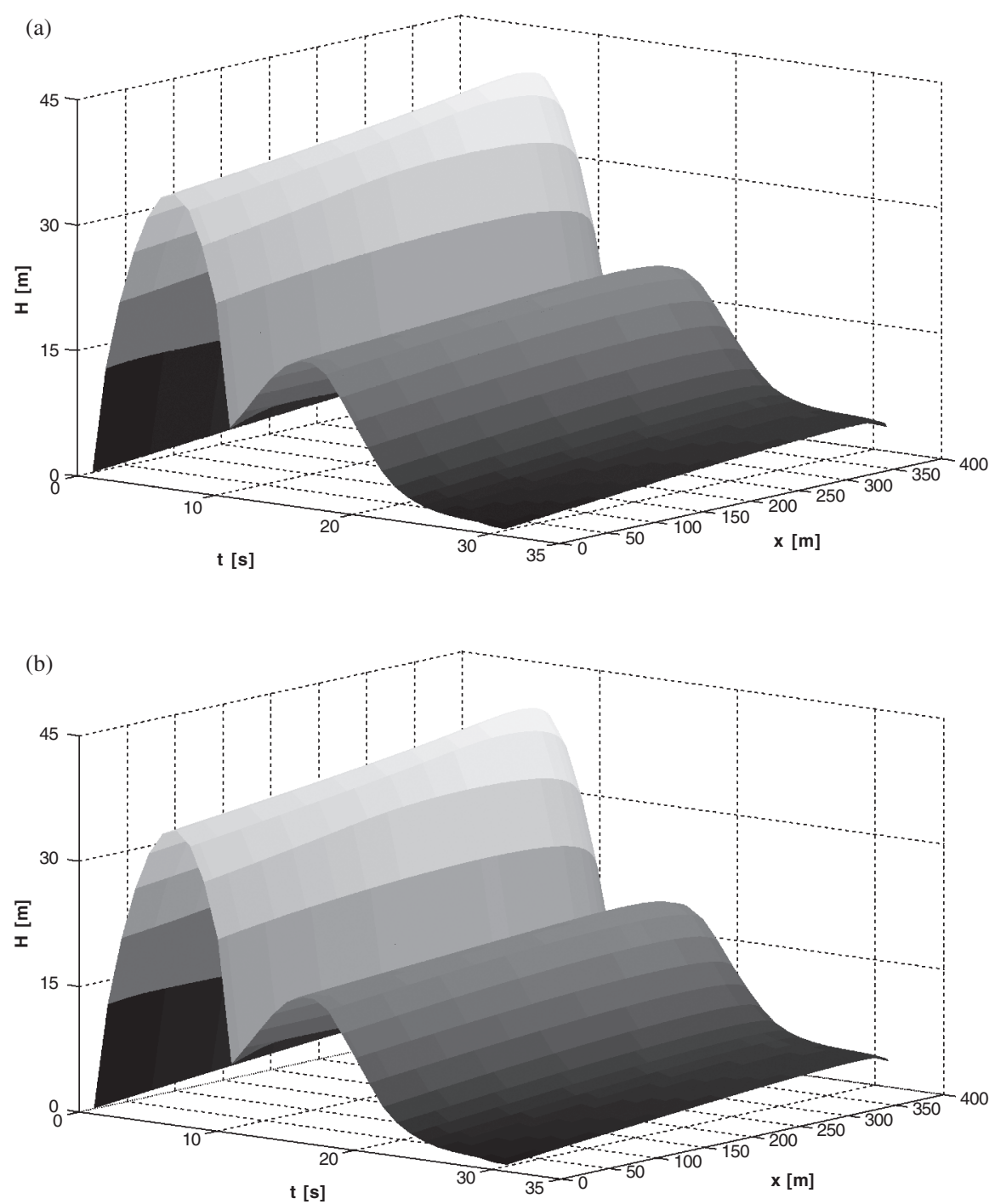

Figure 9: Evolution of the head [m] as a function of the time [s] and the axial position $[\mathrm{m}]$ for the whole pipe: (a) peripheral resistance case; (b) capillary network case.

The pipe is a $10 \mathrm{~m}$ long pipe, with a radius varying from 0.35 to $0.15 \mathrm{~cm}$. The fluid characteristics are $\rho=1,025 \mathrm{~kg} / \mathrm{m}^{3}$ and $\mu=3.2 \mathrm{cP}$ for a haematocrit of $40-45 \%$. The other parameters are the following: $R K_{1}=0.15, R K_{2}=0.45, R K_{3}=0.4, d x=0.025 \mathrm{~m}$.

In both sets of figures, it is clear that the proposed boundary conditions are suitable to represent the phenomenon, the behaviour of the flow being, from a global point of view, similar no matter what the boundary conditions are.

More specifically, at the very downstream position, as can be seen in Fig. $10\left(x=L_{\mathrm{Max}}\right)$, significant differences can be observed. In fact, the numerical value of the terminal pressure influences the behaviour in the case of the peripheral resistance, and not in the case of the capillary network case, which is another advantage of the method. 
(a)

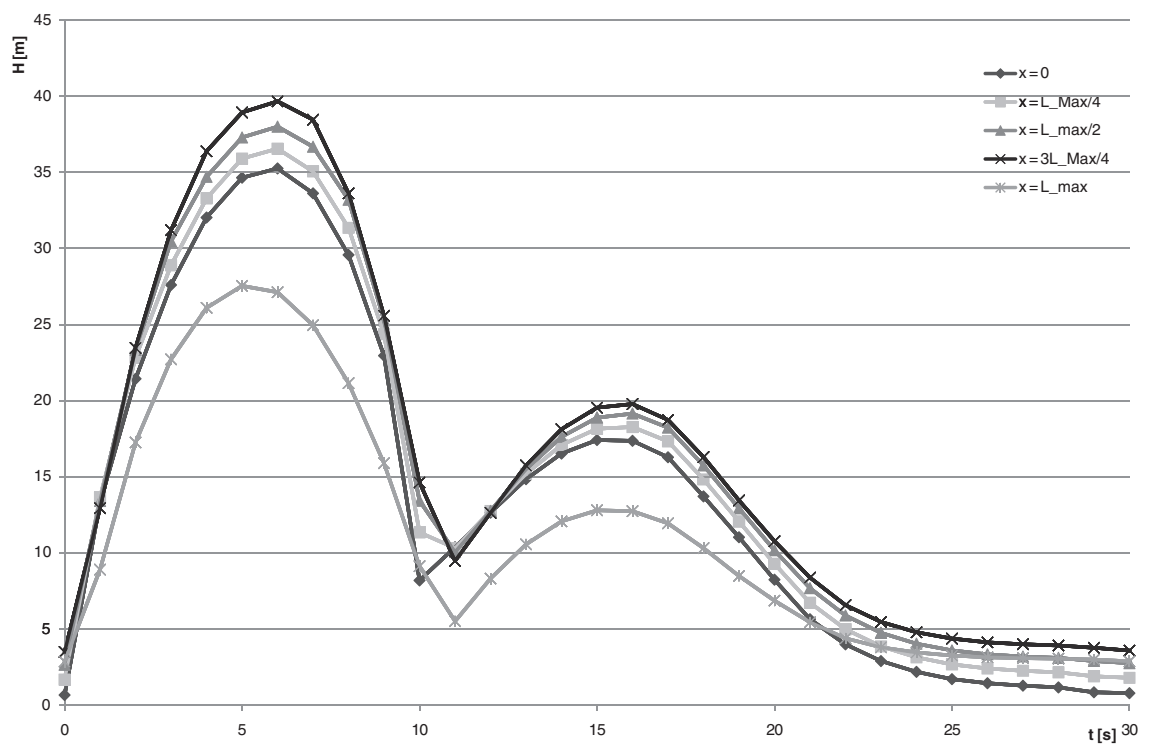

(b)

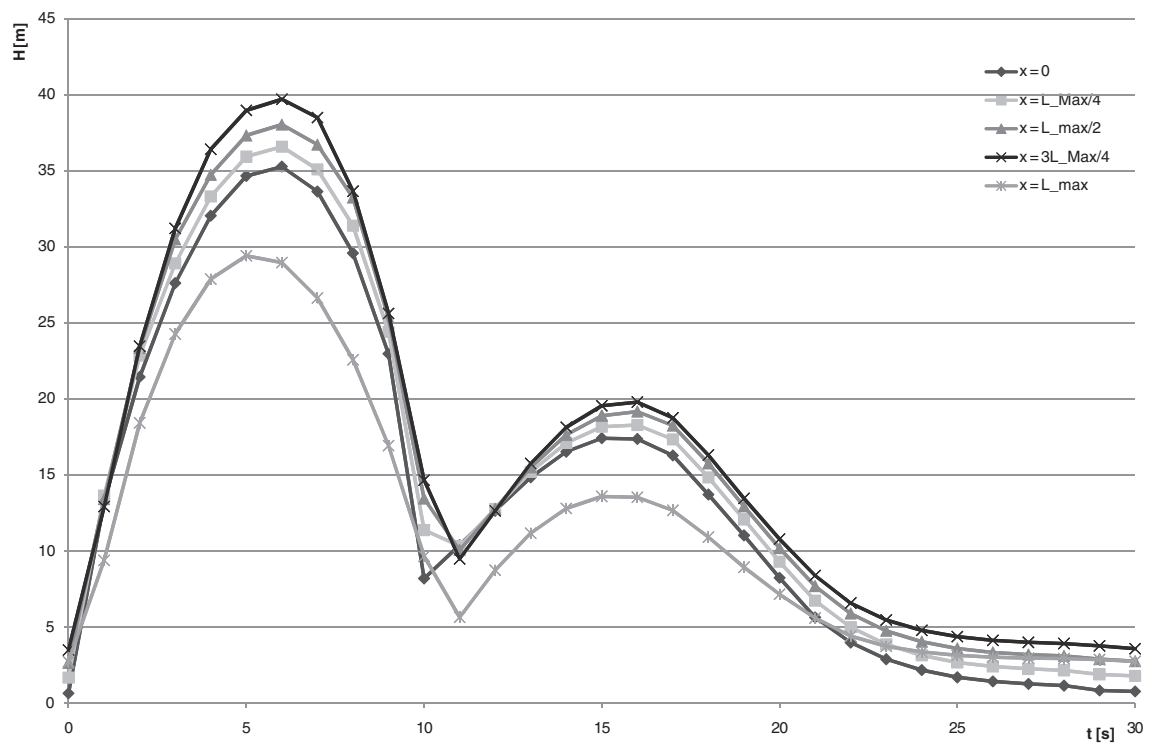

Figure 10: Evolution of the head $[\mathrm{m}]$ as a function of the time [s] for severe axial positions: (a) peripheral resistance case; (b) capillary network case.

\section{CONCLUSIONS}

The presented research for now keeps to the development of the coupled hydraulic model, whereas the final integration of this model with the boundary conditions still remains to be done.

The few results that have been developed and analysed within these researches clearly show interesting tendencies, and also provides information on the physical behaviour of the method. Moreover, 
the work that has already been done indicates clear ways of developments for the final model, which is the last step of the research project.

First, the mechanical analysis of the artery has been done and a mathematical model has been developed such that the implementation of the equations through the final integrated model should be almost direct. Moreover, the numerical model established highlights the sensitivity of the parameters.

Second, a standard 1D flow solver has been implemented using finite volume methods, and enables the modelling of the blood flow through the artery. Although this model does not especially concern blood flow, it is suitable because it handles reliably stiff variation in the flow files, using an original numerical scheme adapted to flows governed by convective effects. Moreover, it enables straightforward modelling at junctions and therefore the physically based simulation of boundary conditions. It has been demonstrated that phenomena such as the dampening of the signal in the capillary network can be reproduced without spurious numerical oscillations. Finally, it has been shown, in a simple case study, that the proposed boundary conditions lead to an accurate prediction of the flow by comparison with classical boundary conditions.

\section{PERSPECTIVES: THE INTEGRATED MODEL}

Now that the different independent parts of the model have all been introduced and developed, the general framework of integration of these models can be described.

It consists of a main model coupled with secondary models and with boundary conditions following the capillary model.

The main model is simply the original hydraulic model presented in Section 4. In the framework depicted in Fig. 12, it is called 'main flow'. The upstream condition requires the prescription of an upstream discharge. The downstream condition refers to the capillary model, as developed in Section 4.4. Basically, depending on the hypothetical presence of capillaries, a certain amount of fluid will be pumped out of the artery and propelled into the capillary following the capillary model.

The presence or lack of capillaries will depend on the geometry chosen at the beginning and obviously on the spatial discretization (see Fig. 11). As shown in Fig. 12, the capillary model uses the modified original main flow model (as explained in Section 4.4 and schematized in Fig. 11), and iteratively evaluates the pressure gradient between the artery and the capillary until the so-called equilibrium capillary discharge is determined.

Two final parameters have to be exploited: the external pressure and the way the fluid is pumped out of the artery have to be considered.

As mentioned in eqns (1-8), the external pressure modifies the cross section of the pipe. Therefore, this parameter somehow influences the original shape of the pipe. The pipe section for an empty slot (noted hereafter in Fig. 12, $\Omega^{*}$ ) and the wave speed will both be modified at each time step in order to represent the external strain.

Besides, the artery/capillary interface is modelled based on a pumping law such that the fluid is taken out of the artery and propelled into the capillary as a function of the pressure difference between the two pipes.

Finally, the model is run as summarized in Fig. 12.

Considering at each time step the new external pressure and re-evaluating the nil-slot parameters, the main flow is evaluated and corrected (or not) due to the presence (or absence) of capillary, and so on, until the information has been propagated in the whole domain.

Finally, many elements, for example, the mechanical parameters of the living tissues (depending on the chosen material model), have to be investigated using experiments. Therefore, the authors plan to conduct a number of parallel researches about anatomical and physiological data, with the purpose of completing and calibrating the models and to finally validate their accuracy. 

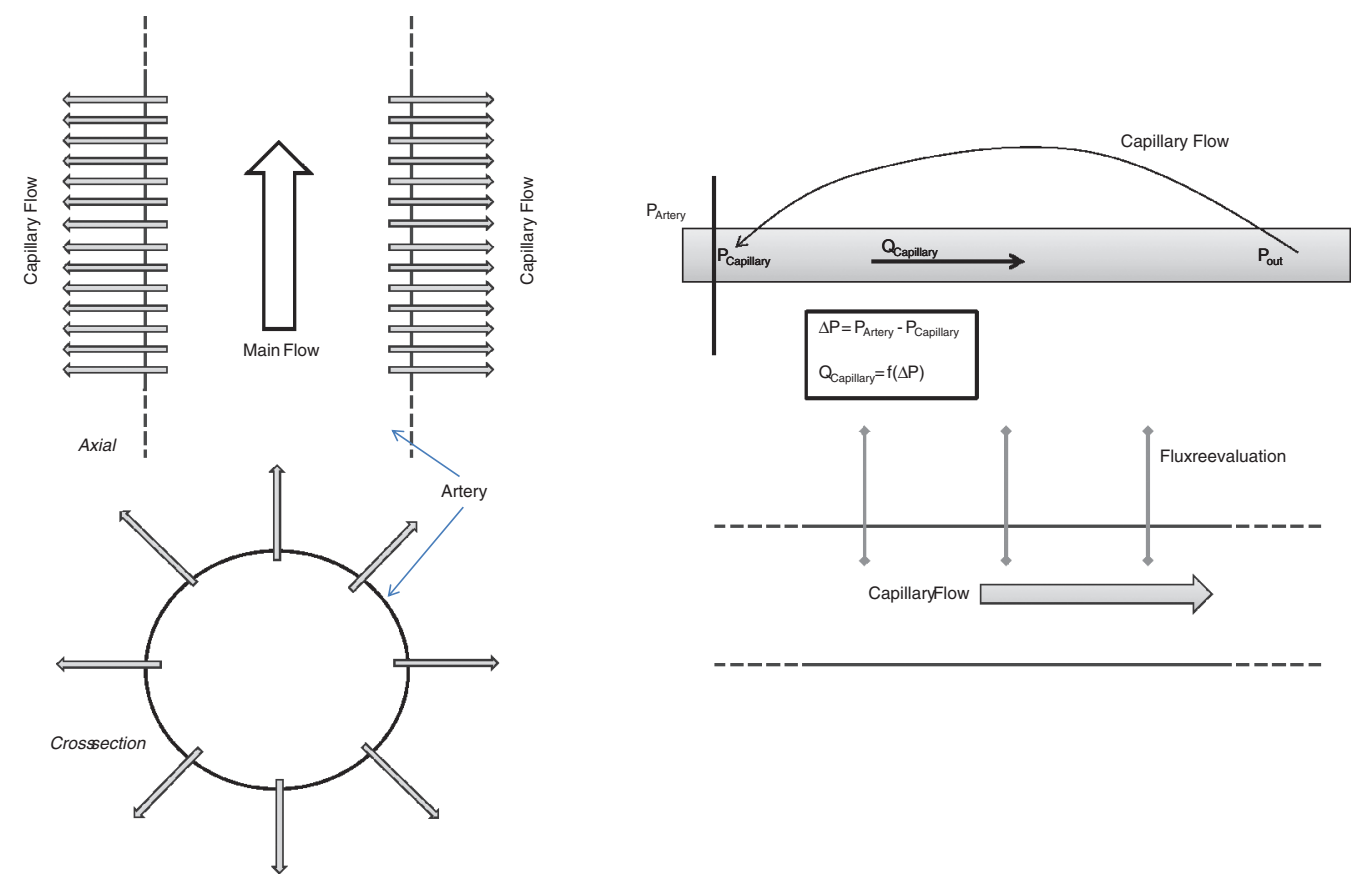

Figure 11: Schematization of the integrated model: (a) global model, (b) artery/capillary interface and (c) capillary model.

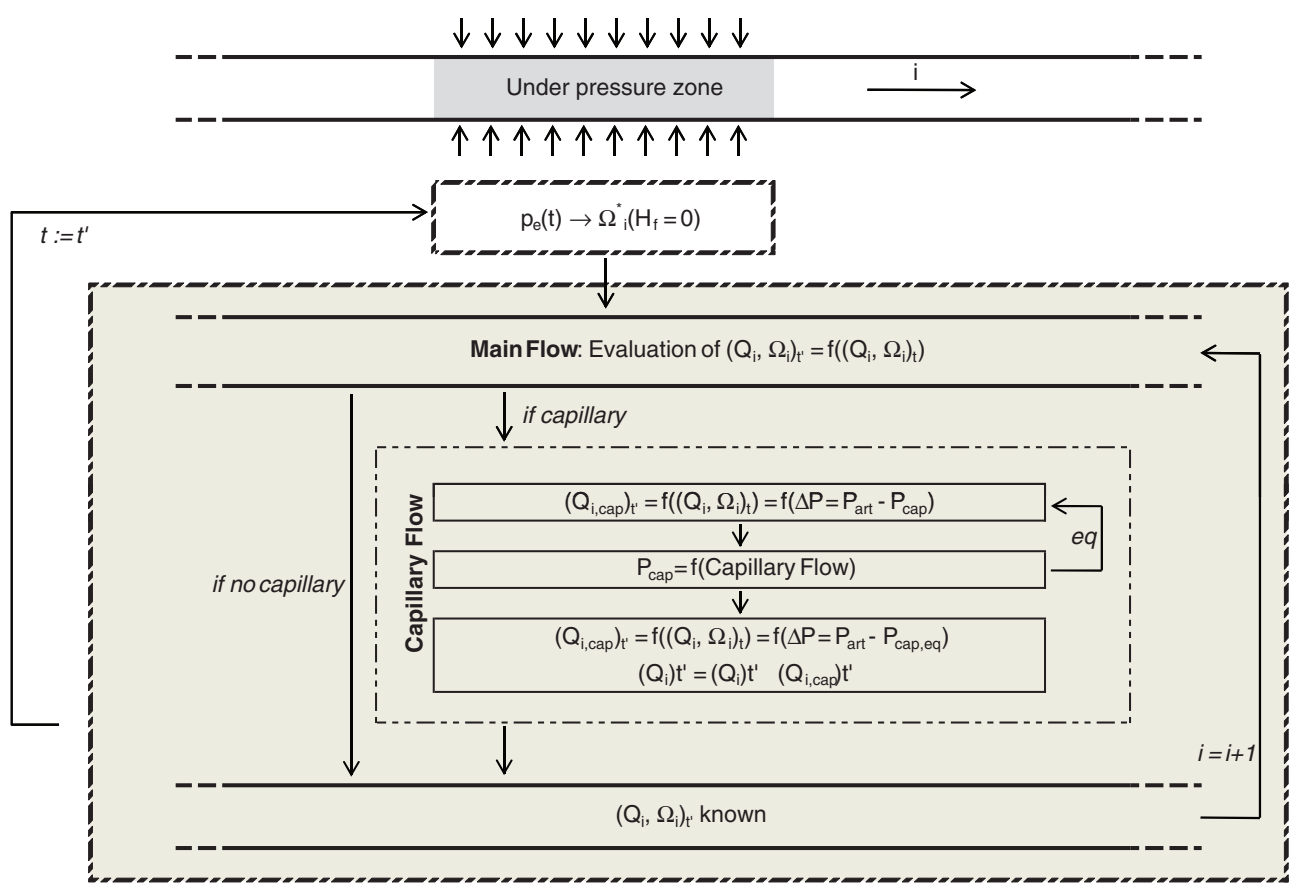

Figure 12: Final model. 


\section{REFERENCES}

[1] Korotkoff, N., On methods of studying blood pressure. Bull Imperial Mil Med, 11, pp. 365-367, 1905.

[2] Riva-Rocci, S., Un nuovo sifigmomanometro. Gazzeta Medica di Torino, 47, pp. 981-996, 1896.

[3] Petrie, J.C., O'Brien, E.T., Littler, W.A. \& de Swiet, M., Recommendations on blood pressure measurement. British Medical Journal, 293, pp. 611-615, 1986.

[4] O'Brien, E., Blood pressure measurement is changing! Hearts, 85-1, p. 3-5, 2001.

[5] O'Brien, E., Fitzgerald, D. \& O'Malley, K., Blood pressure measurement: current practice and future trends. British Medical Journal, 290, pp. 729-734, 1985.

[6] Staessen, J., O'Brien, E., Thijs, L. \& Fagard, R., Modern approaches to blood pressure measurement. Occupational and Environmental Medicine, 57-8, pp. 510-520, 2000. doi:10.1136/oem.57.8.510

[7] Bathe, M., A Fluid-Structure Interaction Finite Element Analysis of Pulsatile Blood Flow through a Compliant Stenotic Artery, PhD Thesis, Department of Mechanical Engineering, Massachusetts Institute of Technology, 1998.

[8] Deparis, S., PhD Thesis, Numerical Analysis of Axisymmetric Flows and Methods for Fluid-Structure Interaction Arising in Blood Flow Simulation, Section de Mathématique, Ecole Polytechnique Fédérale de Lausanne, 2004.

[9] Gerbeau, J.-F., Vidrascu, M. \& Frey, P., Fluid-structure interaction in blood flows on geometries based on medical imaging. Computers \& Structures, 83, pp. 155-165, 2005. doi:10.1016/j. compstruc.2004.03.083

[10] Capron, N., Mompean, G. \& Naji, H., Strong coupling for fluid structure interaction problems. European Journal of Computational Mechanics, 16(3-4), pp. 477-501, 2007.

[11] Yang, J., Preidikman, S. \& Balaras, E., A strong coupling scheme for fluid-structure interaction problems with dynamically moving boundaries in viscous incompressible flows. Mecánica Computacional Vol XXIV, Buenos Aires: Argentina, 2005.

[12] Löhner, R., Cebral, J.R., Chi, Y., Baum, J.D., Meastream, E.L. \& Soto, O., Extending the range and applicability of the loose coupling approach for FSI simulations. Fluid-Structure Interaction; Modelling, Simulation, Optimisation, eds H.-J. Bungartz, M. Schäfer, Springer: Berlin, Heidelberg, pp. 82-100, 2006.

[13] Löhner, R., Cebral, J.R., Chi, Y., Baum, J.D., Mestreau, E., Charman, C. \& Pelessone, D., Large-scale fluid-structure interaction simulations. Computing in Science and Engineering, 6(3), pp. 27-37, 2004. doi:10.1109/MCISE.2004.1289306

[14] Sieber, M.S.G., Numerical Simulation of Fluid-Structure Interaction using Loose Coupling Methods, Numerical Methods in Mechanical Engineering, PhD Thesis, Darmstadt Teknik University, 2001.

[15] Lanoye, L., Swillens, A., Segers, P., Vierendeels, J. \& Verdonck, P., Fluid structure interaction model of an axisymmetrical abdominal aortic aneurysm: decoupled versus fully coupled approach. Advanced Computational Methods in Engineering, Liège, Belgium: LTAS, 2008.

[16] Vierendeels, J., Implicit coupling of partitioned fluid-structure interaction solvers using reduced-order models. Fluid-Structure Interaction; Modelling, Simulation, Optimisation, eds H.-J. Bungartz, M. Schäfer, pp. 1-18, 2006.

[17] Paulus, R., Master Thesis, Analyse des effets mécaniques et hydrauliques induits dans le bras par un brassard de mesure de la pression artérielle, ArGEnCo, ULg, 2007. 
[18] Paulus, R., Dewals, B.J., Erpicum, S., Cescotto, S. \& Pirotton, M., Numerical analysis of coupled mechanical and hydraulic effects induced by a blood pressure meter, 4th Advanced Computational Methods in Engineering, Liège, Belgium: LTAS, 2008.

[19] Paulus, R., Erpicum, S., Dewals, B.J., Cescotto, S. \& Pirotton, M., Computational hemodynamics coupled with mechanical behaviour of the surrounded materials, in the specific case of the brachial artery, 8th International Conference on Modelling in Medicine and Biology, WIT: Crete, Greece, 2009.

[20] Dobrin, P.B., Mechanical properties of arteries. Physiol. Rev., 58(2), pp. 397-460, 1978.

[21] Hayashi, K., Experimental approaches on measuring the mechanical properties and constitutive laws of arterial walls. Journal of Biomechanical Engineering, 115(4B), pp. 481-488, 1993. doi:10.1115/1.2895528

[22] Peterson, L.H., Jensen, R.E. \& Parnell, J., Mechanical properties of arteries in vivo. Circ Res, 8(3), pp. 622-638, 1960.

[23] Pochet, T., Master Thesis, Ecoulement pulsatoire d'un fluide dans une conduite à parois déformables, ULg, 1986.

[24] Fung, Y.C., Biomechanics, Mechanical Properties of Living Tissues, Springer Science: New York, USA, 1993.

[25] Fung, Y.C., Biomechanics, Circulation, Springer Science: New York, USA, 1996.

[26] Archambeau, $\mathrm{P}$., $\mathrm{PhD}$ Thesis, Contribution à la modélisation de la genèse et de la propagation des crues et innondations, ArGEnCo, ULg, 2006.

[27] Preismann, A., Propagation des intumescences dans les canaux et rivières, 1st Congress of the French Association for Computation, Grenoble, France, 1961.

[28] Kerger, F., Archambeau, P., Erpicum, S., Dewals, B.J. \& Pirotton, M., Simulation numérique des écoulements mixtes hautement transitoires dans les conduites d'évacuation des eaux. Houille Blanche-Rev. Int., pp. 159-167, 2009.

[29] Kerger, F., Erpicum, S., Archambeau, P., Dewals, B.J. \& Pirotton, M., Numerical simulation of 1D mixed flow with air/water interaction. Multiphase Flow, New Forest, 2009.

[30] Dewals, B.J., Thesis, Une approche unifiée pour la modélisation d'écoulements à surface libre, de leur effet érosif sur une structure et de leur interaction avec divers constituants, University of Liege, 2006.

[31] Kerger, F., Archambeau, P., Erpicum, S., Dewals, B.J. \& Pirotton, M., A fast universal solver for $1 \mathrm{D}$ continuous and discontinuous steady flows in rivers and pipes. International Journal for Numerical Methods in Fluids, 2009. Published online, doi:10.1002/fld.2243

[32] Levenson, J.A., Peronneau, P.A., Simon, A. \& Safar, M.E., Pulsed Doppler: determination of diameter, blood flow velocity, and volumic flow of brachial artery in man. Cardiovasc Res, 15(3), pp. 164-170, 1981. doi:10.1093/cvr/15.3.164

[33] Morse, O.C. \& Singer, J.R., Blood velocity measurements in intact subjects. Science, 170(3956), pp. 440-441, 1970. doi:10.1126/science.170.3956.440

[34] Anliker, M., Rockwell, R.L. \& Ogden, E., Nonlinear analysis of flow pulses and shock waves in arteries. Zeitschrift für Angewandte Mathematik und Physik (ZAMP), 22(3), pp. 563-581, 1971. doi:10.1007/BF01614000 